Copyright by the IOP PUBLISHING LTD. D. M. Crenshaw et al. 2010 . " radial velocity offsets due to mass outflows and extinction in active galactic nuclei," ApJ 708419 doi:10.1088/0004-637X/708/1/419

The Astrophysical Journal, 708:419-426, 2010 January 1

doi:10.1088/0004-637X/708/1/419

(c) 2010. The American Astronomical Society. All rights reserved. Printed in the U.S.A.

\title{
RADIAL VELOCITY OFFSETS DUE TO MASS OUTFLOWS AND EXTINCTION IN ACTIVE GALACTIC NUCLEI*
}

\author{
D. M. Crenshaw ${ }^{1}$, H. R. Schmitt ${ }^{2,3}$, S. B. Kraemer ${ }^{4}$, R. F. Mushotzky ${ }^{5}$, and J. P. Dunn ${ }^{6}$ \\ ${ }^{1}$ Department of Physics and Astronomy, Georgia State University, Astronomy Offices, One Park Place South SE, Suite 700, Atlanta, GA 30303, USA; \\ crenshaw@chara.gsu.edu \\ ${ }^{2}$ Remote Sensing Division, Naval Research Laboratory, Washington, DC 20375, USA \\ ${ }^{3}$ Interferometrics, Inc., Herndon, VA 20171, USA \\ ${ }^{4}$ Institute for Astrophysics and Computational Sciences, Department of Physics, The Catholic University of America, Washington, DC 20064, USA \\ ${ }^{5}$ NASA/Goddard Space Flight Center, Greenbelt, MD 20771, USA \\ ${ }^{6}$ Department of Physics, Virginia Tech, Blacksburg, VA 24061, USA \\ Received 2009 July 28; accepted 2009 November 6; published 2009 December 11
}

\begin{abstract}
We present a study of the radial velocity offsets between narrow emission lines and host galaxy lines (stellar absorption and H I $21 \mathrm{~cm}$ emission) in Seyfert galaxies with observed redshifts less than 0.043 . We find that $35 \%$ of the Seyferts in the sample show [O III] emission lines with blueshifts with respect to their host galaxies exceeding $50 \mathrm{~km} \mathrm{~s}^{-1}$, whereas only $6 \%$ show redshifts this large, in qualitative agreement with most previous studies. We also find that a greater percentage of Seyfert 1 galaxies show blueshifts than Seyfert 2 galaxies. Using Hubble Spce Talescope/Space Telescope Imaging Spectrograph spatially resolved spectra of the Seyfert 2 galaxy NGC 1068 and the Seyfert 1 galaxy NGC 4151, we generate geometric models of their narrow-line regions (NLRs) and inner galactic disks, and show how these models can explain the blueshifted [O III] emission lines in collapsed STIS spectra of these two Seyferts. We conclude that the combination of mass outflow of ionized gas in the NLR and extinction by dust in the inner disk (primarily in the form of dust spirals) is primarily responsible for the velocity offsets in Seyfert galaxies. More exotic explanations are not needed. We discuss the implications of this result for the velocity offsets found in higher redshift active galactic nuclei.
\end{abstract}

Key words: galaxies: active - galaxies: kinematics and dynamics - galaxies: Seyfert

Online-only material: color figures

\section{INTRODUCTION}

Seyfert galaxies, quasars, and other active galactic nuclei (AGNs) often show radial velocity offsets between their broad emission lines, narrow emission lines, and host galaxy lines (stellar absorption and/or Hi $21 \mathrm{~cm}$ emission), and there is a long history of investigations into the nature of these offsets, beginning with Penston (1977), Netzer (1977), Osterbrock \& Cohen (1979), and Gaskell (1982). In addition, many studies have concentrated on differences in line profile, asymmetry, width (e.g., full-width at half maximum (FWHM)), peak, and/ or velocity centroid as a function of ionization potential or critical density for lines from the same region, such as the narrow-line region (NLR; Osterbrock 1981; Pelat et al. 1981; De Robertis \& Osterbrock 1986; De Robertis \& Shaw 1990; Busco \& Steiner 1992; Moore et al. 1996; Komossa et al. 2008a) or broad-line region (BLR; Wilkes 1984; Crenshaw 1986; Osterbrock \& Mathews 1986; Corbin 1990; Tytler \& Fan 1992; Sulentic et al. 2000; Richards et al. 2002; Snedden $\&$ Gaskell 2007). Even for the same emission line from a low-redshift AGN, such as the commonly used [O III] $\lambda 5007$ line, a perusal of the NASA Extragalactic Database (NED) demonstrates that the heliocentric redshift in terms of radial velocity $(c z)$ can differ substantially in excess of the quoted errors from one study to the next, due to wavelength calibration uncertainties, different measurement techniques, or aperture effects, for example. Thus, some care must be taken when

\footnotetext{
* Based on observations made with the NASA/ESA Hubble Space Telescope, obtained at the Space Telescope Science Institute, which is operated by the Association of Universities for Research in Astronomy, Inc. under NASA contract NAS 5-26555.
}

determining velocity offsets between different lines, but there is no doubt from the above references that the offsets in many sources are real and significant.

Recent investigations have sparked renewed interest in the study of velocity offsets, because they have been used to indicate the possible existence of multiple AGNs in a galaxy, or an AGN that is displaced with respect to the gravitational center of a galaxy. The most convincing evidence comes from galaxies that show active nuclei that are spatially offset from their optical centers, as well as emission lines that are offset in radial velocity from the systemic velocities of the host galaxies. For example, the evidence for multiple AGNs in NGC $3341(z=0.027)$ appears to be incontrovertible (Barth et al. 2008). Three separate nuclei can be identified in a Sloan Digital Sky Survey (SDSS) image of this galaxy. The central nucleus has a LINER/H II composite spectrum, an offset nucleus at a distance of $5.1 \mathrm{kpc}$ from the center has a Seyfert 2 spectrum at a velocity offset of $-200 \mathrm{~km} \mathrm{~s}^{-1}$, and another offset nucleus at a distance of $8.4 \mathrm{kpc}$ has a possible LINER spectrum with a similar velocity offset. Barth et al. suggest that the offset nuclei are due to the fueling of the supermassive black holes (SMBHs) in two dwarf galaxies that are merging with the primary disk galaxy.

Another AGN that has generated a lot of interest and speculation is the quasar SDSS J092712.65+294344.0 $(z=0.71)$, which shows two sets of emission lines (one set of narrow lines and one set of broad plus narrow lines) separated by $2650 \mathrm{~km} \mathrm{~s}^{-1}$ (Komossa et al. 2008b). In this case, the SDSS image shows only an unresolved quasar-multiple nuclei have not been detected in ground-based images. Most of the suggested explanations for the double set of emission lines are based on mergers or interactions of galaxies and, possibly, their SMBHs. Komossa 
et al. (2008b) suggest that the system with broad lines is a recoiling SMBH carrying its BLR and high-ionization NLR with it and leaving behind the bulk of the (lower-ionization) NLR. The recoil is due to coalescence of two black holes which, under the right conditions, can result in anisotropic gravitational radiation that carries away linear momentum, ejecting the merged SMBH at velocities up to $\sim 4000 \mathrm{~km} \mathrm{~s}^{-1}$ in the opposite direction (Campanelli et al. 2007; Baker et al. 2008). Another possible explanation is that this SDSS quasar contains a binary SMBH, in which the BLR and high-ionization NLR are due to accretion onto the smaller SMBH, and the low-ionization NLR is an envelope surrounding the pair of SMBHs (Dotti et al. 2009; Bogdanović et al. 2009). Heckman et al. (2009) suggest a third possibility, which has been used to explain the properties of the nearby AGN NGC 1275: the narrow redshifted lines are associated with a small galaxy falling into the center of a rich cluster of galaxies where it encounters a large galaxy with an AGN that shows both broad and narrow lines. Finally, Shields et al. (2009) suggest that the double set of emission lines in SDSS J092712.65+294344.0 may be due to a chance alignment of two AGNs, possibly within a massive cluster.

Although two sets of emission lines that are offset in radial velocity are rare, many AGNs show velocity offsets between the emission lines from their NLRs and the stellar absorption lines or $\mathrm{H}$ I $21 \mathrm{~cm}$ emission from their host galaxies. Comerford et al. (2009) present a study in which 32 of 91 Seyfert 2 galaxies with red host galaxies at $0.34<z<0.92$ show velocity offsets between their [O III] emission lines and stellar absorption lines that lie in the range $\sim 50 \mathrm{~km} \mathrm{~s}^{-1}$ to $\sim 300 \mathrm{~km} \mathrm{~s}^{-1}$ (two of these AGNs show double peaked emission lines as well). Comerford et al. suggest that the velocity offsets are likely due to displaced AGNs that are moving with respect to their host galaxies. The same explanations for the double set of emission lines in SDSS J092712.65+294344.0 can then be invoked to explain the velocity offsets between the NLR and host galaxy, if one of the AGNs in these scenarios is dispensed with or replaced by an inactive SMBH. However, Comerford et al. (2009) suggest that the most plausible explanation is inspiralling SMBHs after a galaxy merger, which move to the gravitational center of the merger remnant via dynamical friction. They leave open the possibility, however, that the velocity centroids of the emission lines are offset from the systemic velocity of the host galaxy due to a combination of outflows and dust extinction in the NLR, although they say that this is an unlikely explanation for their sample.

In this paper, we show that in low-redshift Seyferts, velocity offsets of the narrow emission lines with respect to the host galaxies are indeed due to mass outflows in the NLR and partial extinction of the outflows by circumnuclear dust. This is not a new idea, as a number of previous studies suggested that the combination of dust, either inside or between the NLR clouds, and radial motion, in the form of inflows or outflows, could be the cause of asymmetries and velocity shifts in the narrow lines (Capriotti et al. 1979; Heckman et al. 1981; Whittle 1985; Dahari \& De Robertis 1988; De Robertis \& Shaw 1990; Veilleux 1991a). Subsequently, a number of studies based on long-slit observations with ground-based telescopes suggested that outflows and dust obscurations could produce the observed profile asymmetries (Storchi-Bergmann et al. 1992; Arribas et al. 1996; Christopoulou et al. 1997; Rodríguez-Ardila et al. 2006). We can now investigate this issue in more detail using spatially resolved optical spectra of the NLR $\left(\sim 0^{\prime \prime} .1\right.$ resolution)

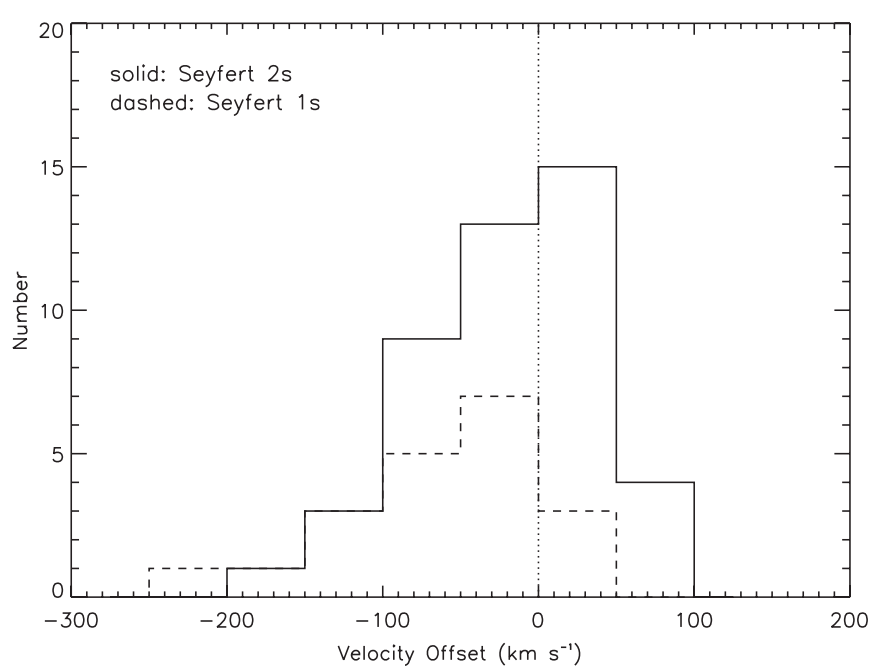

Figure 1. Histograms of the velocity offsets between emission lines and absorption lines $\left(\Delta v=v_{\text {emis }}-v_{\text {abs }}\right)$ in Seyfert 1 and Seyfert 2 galaxies, from the measurements of Nelson \& Whittle (1995).

from the Space Telescope Imaging Spectrograph (STIS) on board the Hubble Space Telescope (HST).

\section{CHARACTERIZATION OF THE VELOCITY OFFSETS}

First, we provide a new characterization of the velocity offsets of the narrow lines in Seyfert galaxies, this time separated by Seyfert type. The best sample to use is that of Nelson \& Whittle (1995), which is based on measurements of moderateresolution $\left(80-230 \mathrm{~km} \mathrm{~s}^{-1}\right)$ ground-based spectra of a large collection of AGNs (mostly Seyfert galaxies) with $z<0.043$. The advantage of this data set, in addition to its size and spectral resolution, is that the observations cover regions in which both strong emission lines and stellar absorption lines are present, reducing possible systematic effects. The blue spectra cover $\mathrm{H} \beta$ and $[\mathrm{O}$ III] $\lambda \lambda 4959,5007$ emission and $\mathrm{Mg} \mathrm{I}$ $\lambda \lambda \lambda 5167,5173,5184$ stellar absorption, and the red spectra cover [S III] $\lambda 9069$ emission and Ca II $\lambda \lambda \lambda 8498,8542,8662$ stellar absorption. Nelson \& Whittle show that their stellar velocities, combined from the $\mathrm{Mg}$ I and Ca II triplets, are in agreement with the published $\mathrm{H}$ I $21 \mathrm{~cm}$ velocities to within the published uncertainties.

From Table 4 in Nelson \& Whittle (1995), we adopted the stellar absorption velocity $\left(v_{\text {abs }}\right)$ and used the [O III] emission velocity centroid $\left(v_{\mathrm{em}}\right)$ for each AGN to determine the velocity offset: $\Delta v=v_{\text {emis }}-v_{\text {abs }}$. In the few cases, where [O III] velocities were not listed, we used [S III] velocities instead. The mean uncertainties of the sample are $15 \mathrm{~km} \mathrm{~s}^{-1}$ in $v_{\text {abs }}$ and $7 \mathrm{~km} \mathrm{~s}^{-1}$ in $v_{\mathrm{em}}$, which added in quadrature yield an uncertainty in $\Delta v$ of $17 \mathrm{~km} \mathrm{~s}^{-1}$. To be conservative, we consider an offset $\gtrsim 3 \sigma$, i.e., $|\Delta v| \geqslant 50 \mathrm{~km} \mathrm{~s}^{-1}$, to be significant. We identified the AGNs in this data set that are Seyfert galaxies and determined their types using information in NED (placing Seyfert 1.8s and 1.9s into the type 2 category). This yielded a total of 65 Seyfert 1 or 2 galaxies with velocity offsets determined in a uniform manner.

In Figure 1, we plot histograms of the velocity offsets in $50 \mathrm{~km} \mathrm{~s}^{-1}$ bins. The distributions of both Seyfert types peak near $0 \mathrm{~km} \mathrm{~s}^{-1}$, but both show an extended tail on the blueshifted side that goes up to -200 to $-250 \mathrm{~km} \mathrm{~s}^{-1}$. Combining the two histograms, $23 / 65$ (35\%) of the Seyferts show blueshifted emission lines with $\Delta v \leqslant-50 \mathrm{~km} \mathrm{~s}^{-1}$, whereas $4 / 65(6 \%)$ of the Seyferts show redshifted lines with $\Delta v \geqslant+50 \mathrm{~km} \mathrm{~s}^{-1}$. 
These results are in agreement with many other studies, which find that the [O III] lines in low-redshift AGNs often have blue asymmetries (more flux in the blue side of the profile) and/or blueshifted peaks or centroids with respect to their systemic velocities, whereas few show red asymmetries or redshifted velocity offsets (Heckman et al. 1981; Whittle 1985; De Robertis \& Shaw 1990; Veilleux 1991b; Bian et al. 2005; Boroson 2005; Komossa et al. 2008a). One exception is the study of Vrtilek \& Carleton (1985), which finds a symmetric distribution of velocity offsets between emission and absorption lines, but their results depend on only 13 Seyfert galaxies and low-redshift quasi-stellar objects.

The Seyfert 1 distribution shown in Figure 1 is shifted to more negative offset velocities (higher blueshifts, on average) than the Seyfert 2 distribution. A Kolmogorov-Smirnov test on the velocity offsets for the two types gives only a $2.1 \%$ probability that two samples from the same population would differ by this much, indicating that the distributions are indeed different. Although this result is based on only 20 Seyfert $1 \mathrm{~s}$ and studies of larger samples are needed to test it, it suggests an additional constraint on possible explanations or models of the velocity offsets.

\section{HST/STIS CONSTRAINTS ON THE NLR KINEMATICS AND GEOMETRY}

As mentioned in the introduction, a number of previous studies have suggested that velocity offsets and/or asymmetries of the narrow emission lines could be produced by a combination of radial motion and dust extinction in the NLR. We can test this notion using HST/STIS long-slit spectra. We have found that that the kinematics of the NLR are dominated by radial outflow in the four nearby Seyferts that we have studied to date: NGC 4151 (Hutchings et al. 1998; Crenshaw et al. 2000; Das et al. 2005), NGC 1068 (Crenshaw \& Kraemer 2000; Das et al. 2006, 2007; see also Cecil et al. 2002), Mrk 3 (Ruiz et al. 2001), and Mrk 573 (T. Fischer et al. 2010, in preparation, see also Schlesinger et al. 2009). Our kinematic models of biconical outflow provide a good match to the overall flow patterns as a function of distance from the central AGN, despite significant local variations in the velocity fields. But the main arguments for radial outflow are independent of our detailed models. The velocities of the bright NLR clouds (or knots) close to the nucleus reach values that are too high to be explained by any sort of reasonable gravitational potential (including contributions from the SMBH, nuclear stellar cluster, and bulge; Das et al. 2007), which rule out infall or orbital motions as being dominant. Transverse motions of the gas away from the radio jets (Axon et al. 1998; Capetti et al. 1999) cannot be dominant, because they cannot explain NLRs with primarily blueshifts from one cone and redshifts from the other (as in NGC 4151, Crenshaw \& Kraemer 2000). Only radial outflow can match the kinematics of the Seyfert NLRs that we have studied to date. Furthermore, radial outflow is consistent with the unified model of Seyfert galaxies (Antonucci 1993), which postulates that an optically thick torus outside of the BLR produces a bicone of radiation that ionizes the NLR and that Seyfert type depends on viewing angle with respect to the bicone axis. The three Seyfert 2 galaxies studied to date show blueshifts and redshifts on either side of the central AGN, consistent with the unified model prediction of a bicone axis near the plane of the sky, whereas the Seyfert 1.5 galaxy NGC 4151 shows blueshifts on one side and redshifts on the other side of the central AGN, consistent with an intermediate viewing angle (Crenshaw et al. 2000). A

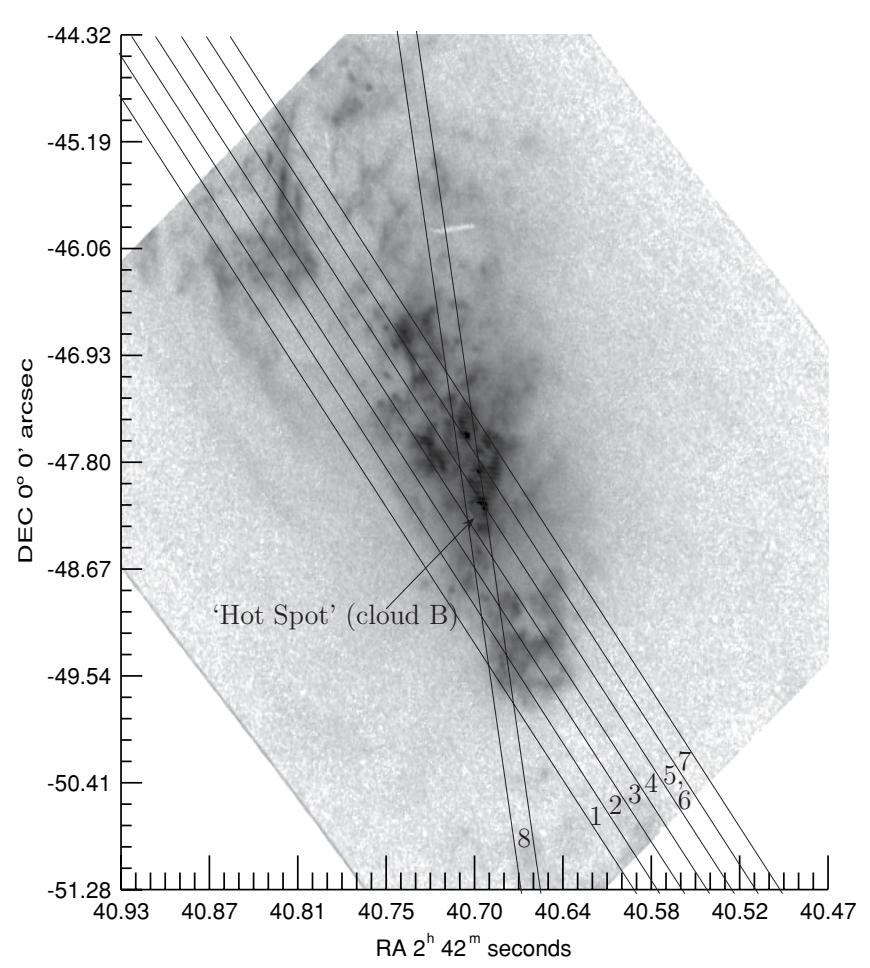

Figure 2. [O III] emission-line image of the NLR in NGC 1068, obtained with the HST Faint Object Camera, showing the slit positions for the STIS long-slit observations with the G430M grating. The SMBH is 0.15 south of the hot spot (Das et al. 2006).

number of recent studies based on ground-based observations with adaptive optics and field spectrographs have also found evidence for radial outflows in the NLRs of Seyfert galaxies (Barbosa et al. 2009; Riffel et al. 2009; Stoklasová et al. 2009; Storchi-Bergmann et al. 2009).

\subsection{Simulation of Ground-Based Spectra}

In order to compare our results with those from previous ground-based spectra, which were obtained through apertures that include most or all of the NLRs in even the closest Seyferts (only a few arcseconds in extent), we use HST/STIS long-slit spectra that cover most of the NLR as well. The two data sets that meet this requirement are the STIS observations of NGC 1068 (Cecil et al. 2002; Das et al. 2006) and NGC 4151 (Das et al. 2005) obtained at multiple parallel slit positions, as shown in Figures 2 and 3. Spectra were obtained with the G430M grating through the $52^{\prime \prime} \times 0^{\prime \prime} .2$ slit at a spectral resolving power of $R=$ $\lambda / \Delta \lambda \approx 9000$ and an angular resolution of $\sim 0^{\prime \prime} .1$ in the crossdispersion direction. This procedure resulted in hundreds of spectra of the $\mathrm{H} \beta$ and $[\mathrm{O} \mathrm{III}] \lambda \lambda 4959,5007$ lines along the slits. As shown in Figures 2 and 3, the NLRs are dominated by emission-line knots that are resolved and on the order of a few tenths of an arcsecond in size; we found that each knot has a unique radial velocity and a fairly large velocity dispersion, on the order of hundreds of $\mathrm{km} \mathrm{s}^{-1}$ FWHM (Das et al. 2005, 2006).

To simulate the ground-based spectra, we added together all of the spectra along each slit position, and then added together the spectra from each slit (positions 6 and 8 in NGC 1068 were excluded because they overlap the other slit positions). In Figures 4 and 5, we give these "collapsed" spectra in terms of flux as a function of radial velocity of the [O III] $\lambda 5007$ line, with respect to the rest frame established by observations of H I $21 \mathrm{~cm}$ emission in each galaxy. The unsmoothed profiles 


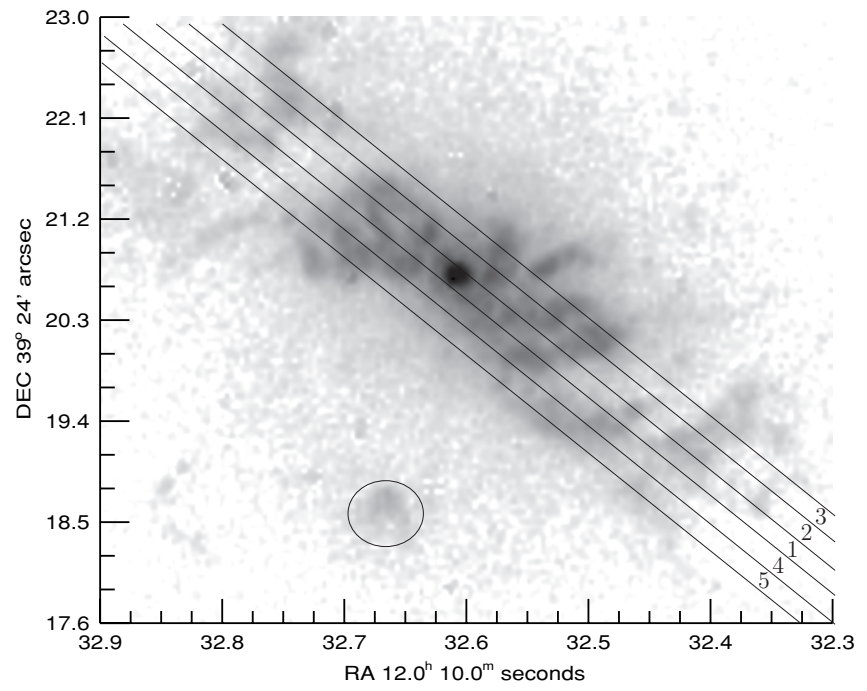

Figure 3. [O III] emission-line image of the NLR in NGC 4151, obtained with the HST Wide Field Planetary Camera 2, show the slit positions for the STIS G430M long-slit observations (see also Das et al. 2005). The circled feature is due to a reflection in the camera.

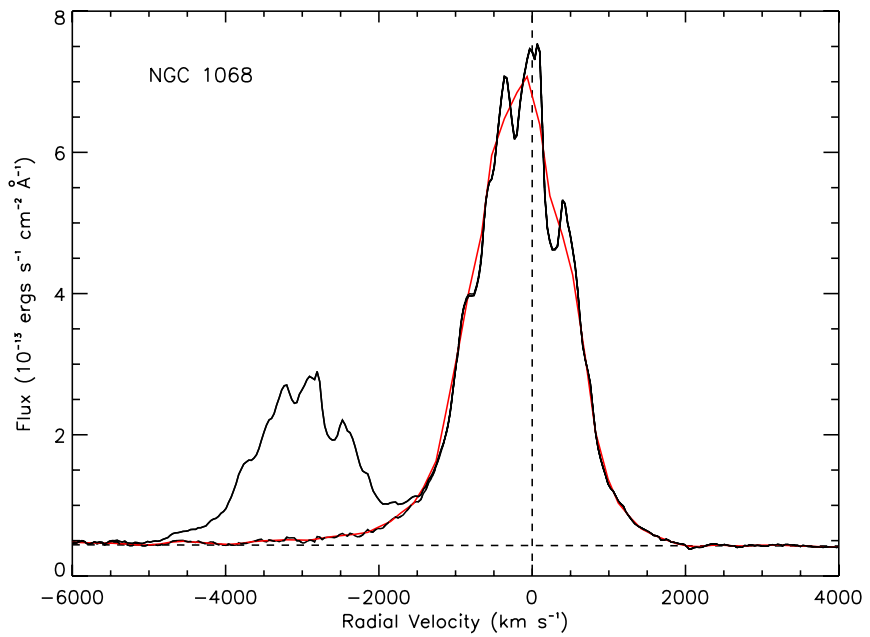

Figure 4. Collapsed spectra of the [O III] emission lines in NGC 1068, obtained by adding together all of the spectra from the STIS long-slit observations and plotting the flux vs. radial velocity, where $0 \mathrm{~km} \mathrm{~s}^{-1}$ is defined by $\mathrm{H}_{\mathrm{I}} 21 \mathrm{~cm}$ emission. The solid black lines shows the unsmoothed $(\mathrm{R} \approx 9000)$ spectrum, and the deblended blue wing of [O III] $\lambda 5007$ underneath the [O III $] \lambda 4959$ line. The solid red line shows the heavily smoothed $(R \approx 1000)$ version of the [O III] $\lambda 5007$ line.

(A color version of this figure is available in the online journal.)

in Figures 4 and 5 show significant structure with multiple peaks. In fact, they are virtually identical to the [O III] profiles from the high-resolution ground-based spectra of Vrtilek \& Carleton (1985) and Veilleux (1991c), giving us confidence that any [O III] emission missed by the STIS long-slit observations does not significantly alter the profile structure or shape. From our analysis of the resolved STIS spectra, it is clear that this structure is due to the superposition of velocity profiles from the numerous emission-line knots seen in Figures 2 and 3, with each knot having its own peculiar radial velocity with respect to the general outflow pattern (Das et al. 2005, 2006).

The [O III] lines in NGC 1068 are much broader than those in NGC $4151\left(\mathrm{FWHM}=1450[ \pm 15] \mathrm{km} \mathrm{s}^{-1}\right.$ and $200[ \pm 10] \mathrm{km}$ $\mathrm{s}^{-1}$, respectively), consistent with the overall higher velocities and dispersions of the emission-line knots in NGC 1068. The [O III] lines in NGC 1068 are blended together in their wings,

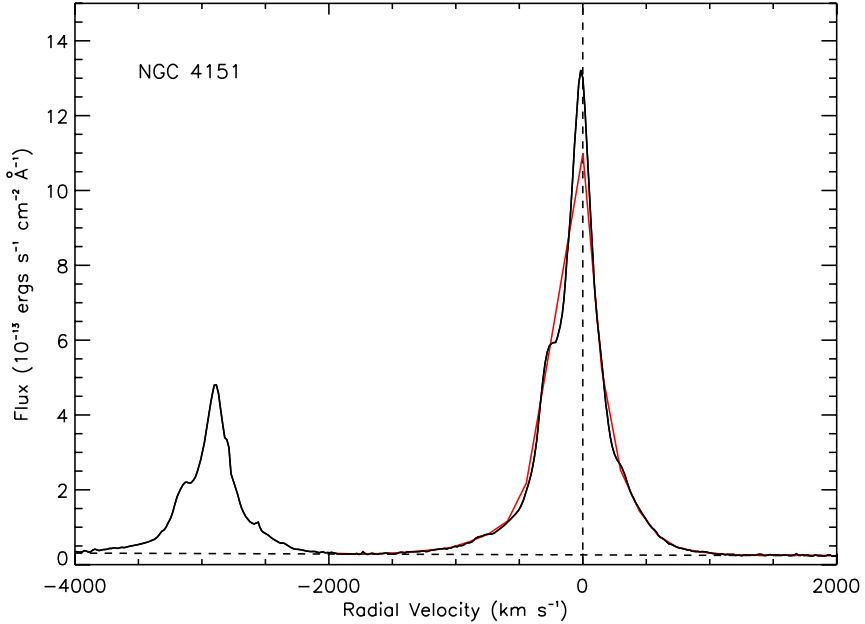

Figure 5. Collapsed spectra of the [O III] emission lines in NGC 4151, obtained by adding together all of the spectra from the STIS long-slit observations and plotting the flux vs. radial velocity, where $0 \mathrm{~km} \mathrm{~s}^{-1}$ is defined by H $21 \mathrm{~cm}$ emission. The solid black line shows the unsmoothed $(R \approx 9000)$ spectrum The solid red line shows the heavily smoothed $(R \approx 1000)$ version of the [O III] $\lambda \lambda 4959,5007$ lines.

(A color version of this figure is available in the online journal.)

Table 1

Heliocentric Radial Velocities and Offsets (in $\mathrm{km} \mathrm{s}^{-1}$ )

\begin{tabular}{lrrrrr}
\hline \hline $\begin{array}{l}\text { Hi } 21 \mathrm{~cm} \\
\text { Emission }\end{array}$ & $\begin{array}{c}\text { Stellar } \\
\text { Absorption }\end{array}$ & \multicolumn{2}{c}{ Centroid } & \multicolumn{1}{c}{ Peak } & \multicolumn{2}{c}{ Centroid } & \multicolumn{1}{c}{ Peak } \\
\hline \multicolumn{5}{c}{ NGC 1068 } \\
$1137 \pm 3^{\mathrm{a}}$ & $1149 \pm 8^{\mathrm{b}}$ & $974 \pm 7$ & $1128 \pm 5$ & $962 \pm 8$ & $988 \pm 7$ \\
$\Delta v$ & $12 \pm 9$ & $-163 \pm 8$ & $-9 \pm 6$ & $-175 \pm 9$ & $-149 \pm 8$ \\
\hline \multicolumn{5}{c}{ NGC 4151} \\
$995 \pm 3^{\mathrm{c}}$ & $1006 \pm 18^{\mathrm{b}}$ & $935 \pm 5$ & $998 \pm 4$ & $926 \pm 3$ & $981 \pm 4$ \\
$\Delta v$ & $11 \pm 18$ & $-60 \pm 6$ & $3 \pm 5$ & $-69 \pm 4$ & $-14 \pm 5$ \\
\hline
\end{tabular}

Notes.

a Bottinelli et al. 1990 .

b Nelson \& Whittle 1995.

c de Vaucouleurs et al. 1991.

and we deblended the two lines by taking the $\lambda 5007$ profile, reproducing it at the position of the $\lambda 4959$ line, scaling it by one-third (the ratio of the transition probabilities of the two lines), subtracting it from the blend, and repeating the process in an iterative fashion. To simulate low-resolution ground-based spectra at $R=1000$, we convolved the unsmoothed spectra with a Gaussian with a FWHM $=500 \mathrm{~km} \mathrm{~s}^{-1}$ (representing the line-spread function) and rebinned them to one-half of a resolution element. These "low-resolution spectra" are also shown in Figures 4 and 5.

The [O III] profiles in Figures 4 and 5 are clearly asymmetric, with more flux in the blue sides of the profiles. To quantify the velocity offsets, we measured the velocity centroid of the [O III] $\lambda 5007$ line (averaged over the entire profile) and its velocity peak (defined as the centroid averaged over the top $15 \%$ of the [O III] line). In Table 1, we give the heliocentric velocities from the H $21 \mathrm{~cm}$ emission, the stellar absorption lines, and the [O III] measurements described above, as well as the velocity offsets relative to the $\mathrm{H}_{\mathrm{I}} 21-\mathrm{cm}$ velocity.

From Table 1, it is clear that the $\mathrm{H}_{\mathrm{i}} 21 \mathrm{~cm}$ emission and the stellar absorption features give the same heliocentric velocities, to within the measurement errors of $\sim 10 \mathrm{~km} \mathrm{~s}^{-1}$. This gives us confidence that the systemic velocities of the host galaxies are well established. For the [O III] measurements, the velocity 
centroids are the same to within the errors in both high and low resolution spectra for each galaxy, whereas the velocity peaks are discrepant, particularly for NGC 1068. Thus, it is clear that the velocity centroids provide a much more reliable measure of the velocity offsets. This would be especially true for lower signal-to-noise spectra, where random variations could alter the location of the peak emission significantly.

Looking at the high resolution spectra, the velocity centroids of the [O III] emission are blueshifted by $-160 \mathrm{~km} \mathrm{~s}^{-1}$ in NGC 1068 and $-60 \mathrm{~km} \mathrm{~s}^{-1}$ in NGC 4151. These fall inside of the range of values for Seyfert galaxies shown in Figure 1, and are what we consider to be significant offsets $(|\Delta v|>50 \mathrm{~km}$ $\mathrm{s}^{-1}$ ). Interestingly, the velocity offset for NGC 1068, a Seyfert 2 , is greater in magnitude than that for NGC 4151, a Seyfert 1 galaxy. This is likely due to a combination of higher velocities and higher extinction in NGC 1068 (Das et al. 2005, 2006), which we discuss further in the next section.

\subsection{Geometry of the Outflows and Extinction}

To investigate the nature of extinction in the NLR, we first review our previous studies using $H S T$ /STIS long-slit, lowresolution $(R \approx 1000) \mathrm{UV}$ and optical spectra of NGC 1068 (Kraemer \& Crenshaw 2000) and NGC 4151 (Kraemer et al. 2000). The reddening of the narrow emission lines in these Seyferts, based on the He II $\lambda 1640 / \lambda 4686$ ratio, varies between $E(B-V)=0.1$ and 0.5 in a nonuniform fashion across their NLRs. Based on our photoionization models, the reddening cannot come primarily from dust in the ionized gas, because the NLR clouds contains either no dust or a reduced dust fraction (between $10 \%$ and 50\%) compared to the normal dust/gas ratio in the local ISM of our Galaxy; otherwise, lines from the refractory elements (such as $\mathrm{Mg}, \mathrm{Si}$, and $\mathrm{Fe}$ ) would be much weaker than observed, and Ly $\alpha$ emission would be quenched, due to multiple resonant scatterings and eventual absorption by dust grains. Even the NLR clouds with a $50 \%$ dust fraction cannot produce the observed reddening, since they have a hydrogen column $N_{H}<10^{21} \mathrm{~cm}^{-2}$, which would produce a reddening of $E(B-V)<0.1$ (assuming the Galactic reddening law of Savage \& Mathis 1979 and $N_{H}=5.2 \times 10^{21} E(B-V)$ $\mathrm{cm}^{-2}$ from Shull \& van Steenberg 1985). Thus, the reddening is likely produced in patchy dust that is external to the NLR clouds. Circumnuclear dust spirals seen in the inner disks of most Seyfert galaxies (Malkan et al. 1998; Regan \& Mulchaey 1999; Pogge \& Martini 2002, Martini et al. 2003; Deo et al. 2006) are the likely source of the reddening; they extend over the same scales as the NLR (hundreds of parsecs) and the dust spirals would provide the observed patchiness.

To investigate the effects of extinction on mass outflows in the NLR, we therefore assume that the reddening arises from dust in the inner disk of the host galaxy. In order to determine the three-dimensional geometry of the outflows and extinction, we combined our parameters from the biconical outflow models with the observed parameters of the host galaxy disks. In Table 2, we give the five required parameters from Das et al. (2005, 2006): the position angle (P.A.) of the galaxy's major axis, the inclination $(i)$ of the galactic disk (zero corresponds to face-on), the P.A. of the bicone axis, the inclination of the bicone axis (zero is in the plane of the sky), and the half-opening angle (H.O.A.) of the outer edge of the bicone. For the inclinations, we also indicate which side of the structure is closest to us.

In Figure 6, we show the geometry of the biconical outflow and the host galaxy disk for NGC 1068 and NGC 4151. For simplicity, we depict the midplane of the galaxy and the outer
Table 2

NLR Bicone and Host Galaxy Parameters ${ }^{\mathrm{a}}$

\begin{tabular}{lccc}
\hline \hline \multicolumn{1}{c}{ Parameter } & NGC 1068 & NGC 4151 & Test \\
\hline P.A. (Galaxy) & $106^{\circ}$ & $22^{\circ}$ & $90^{\circ}$ \\
Incl. (Galaxy) & $40^{\circ}(\mathrm{SW})$ & $20^{\circ}(\mathrm{W})$ & $60^{\circ}(\mathrm{S})$ \\
P.A. (Bicone) & $30^{\circ}$ & $60^{\circ}$ & $0^{\circ}$ \\
Inclin. (Bicone) & $5^{\circ}(\mathrm{NE})$ & $45^{\circ}(\mathrm{SW})$ & $30^{\circ}(\mathrm{S})$ \\
H.O.A. (Bicone) & $40^{\circ}$ & $33^{\circ}$ & $30^{\circ}$ \\
\hline
\end{tabular}

Notes. ${ }^{a}$ See the text for a description of the parameters, obtained from Das et al. $(2005,2006)$ for NGC 1068 and NGC 4151. The letters in parentheses indicate the side closest to us.

surface of the bicone, even though the bicone extends to an inner opening angle in our kinematic models. The left-hand side shows the view from Earth, and the right-hand side shows a viewpoint in which the bicone axis is in the plane of the sky and the galactic disk is edge-on, to show the true angle between the bicone and the disk (see the figure captions for more details). The parallel lines on either side of the galactic midplane show the dust scale height if we assume a typical value of $\sim 200 \mathrm{pc}$ for spiral galaxies (Xilouris et al. 1999) and a length of $\sim 800 \mathrm{pc}$ for each bicone along its axis (Das et al. 2005, 2006).

In the case of NGC 1068, the SW side of the galaxy is closer to us, and the SW cone therefore experiences more extinction. This is consistent with the [O III] image in Figure 2, which shows weaker, and in some place absent, emission SW of the location of the SMBH, which is 0 ".15 south of the "hot spot" in Figure 2 (Das et al. 2006). The bicone axis lies nearly in the plane of the sky and the nearer side of each cone is blueshifted, whereas the farther side of each cone is redshifted. Because the disk has a finite thickness, the blueshifted side of each cone in NGC 1068 experiences less extinction, on average, than the redshifted side of each cone. In the case of NGC 4151, the SW cone is entirely blueshifted and the NE cone is entirely redshifted. The host galaxy disk is close to the plane of the sky, and the SW cone shows less extinction than the NE cone shows. Thus, both geometries lead to emission-line profiles that are asymmetric to the blue, in agreement with the observed profiles.

To determine if this explanation makes quantitative sense, we can look at the asymmetry of the emission-line profiles, and ask if there is enough dust in the inner galactic disks to produce the asymmetries. For simplicity, let us assume that the blue wings of the profiles are not affected by extinction and that the red wings are affected by a simple screen. In this case, the ratios of blue-to-red wing fluxes for [O III] $\lambda 5007$ are 1.59 for NGC 1068 and 1.10 for NGC 4151, which yield extinctions in the $V$ band of $A_{V}=0.5 \mathrm{mag}$ and $0.1 \mathrm{mag}$, respectively. These are likely underestimates of the total extinction because the blue wings are almost certainly affected by extinction as well, as discussed above. From a study of spiral galaxy disks, Xilouris et al. (1999) found that the central extinctions ranged from $A_{V}=0.3 \mathrm{mag}$ to 0.8 mag. In a study of nuclear dust spirals, Martini \& Pogge (1999) determined color excesses between arm and interarm regions that correspond to extinctions in the range $A_{V}=0.4$ mag to $1.4 \mathrm{mag}$, assuming a standard Galactic reddening curve (Savage \& Mathis 1979). Our STIS spectra yield reddening values from the He II $\lambda 1640 / \lambda 4686$ line ratio that correspond to $A_{V}=0.3 \mathrm{mag}$ to $1.5 \mathrm{mag}$ (Kraemer \& Crenshaw 2000; Kraemer et al. 2000). Thus, based on the above simple calculation, there is sufficient dust in the inner galactic disk to account for the differences in observed flux between the red and blue wings of the profiles. 

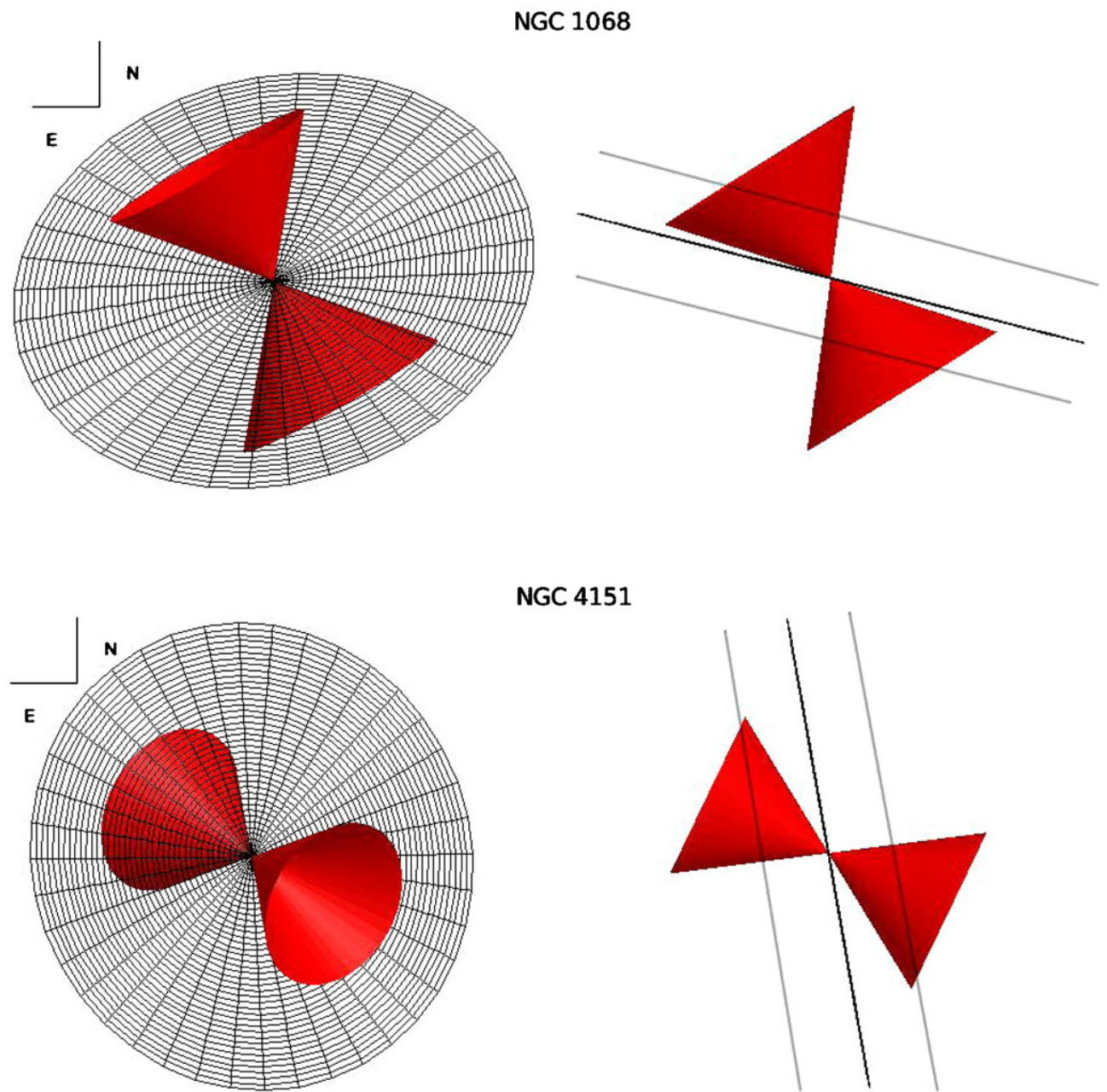

\section{NGC 4151}

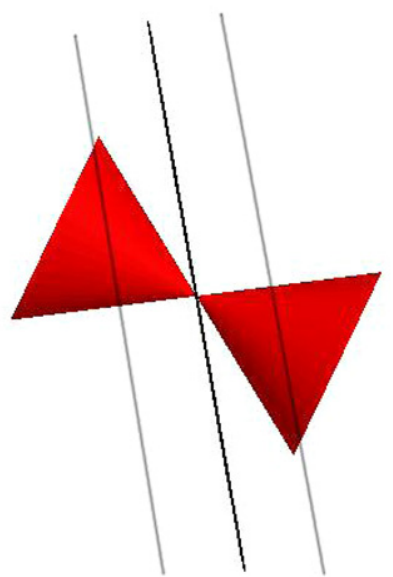

Figure 6. Geometric models of the NLR bicones and the inner galactic disks in NGC 1068 and NGC 4151, based on the parameters in Table 2 . The left-hand side shows our view, and the right hand side shows a view in which the bicone axis is in the plane of the sky and the galactic disk is edge-on (our view is to the upper right and above the page for each). The thin gray lines represent a disk scale height of $200 \mathrm{pc}$ for a bicone that is $800 \mathrm{pc}$ in length along its axis.

(A color version of this figure is available in the online journal.)
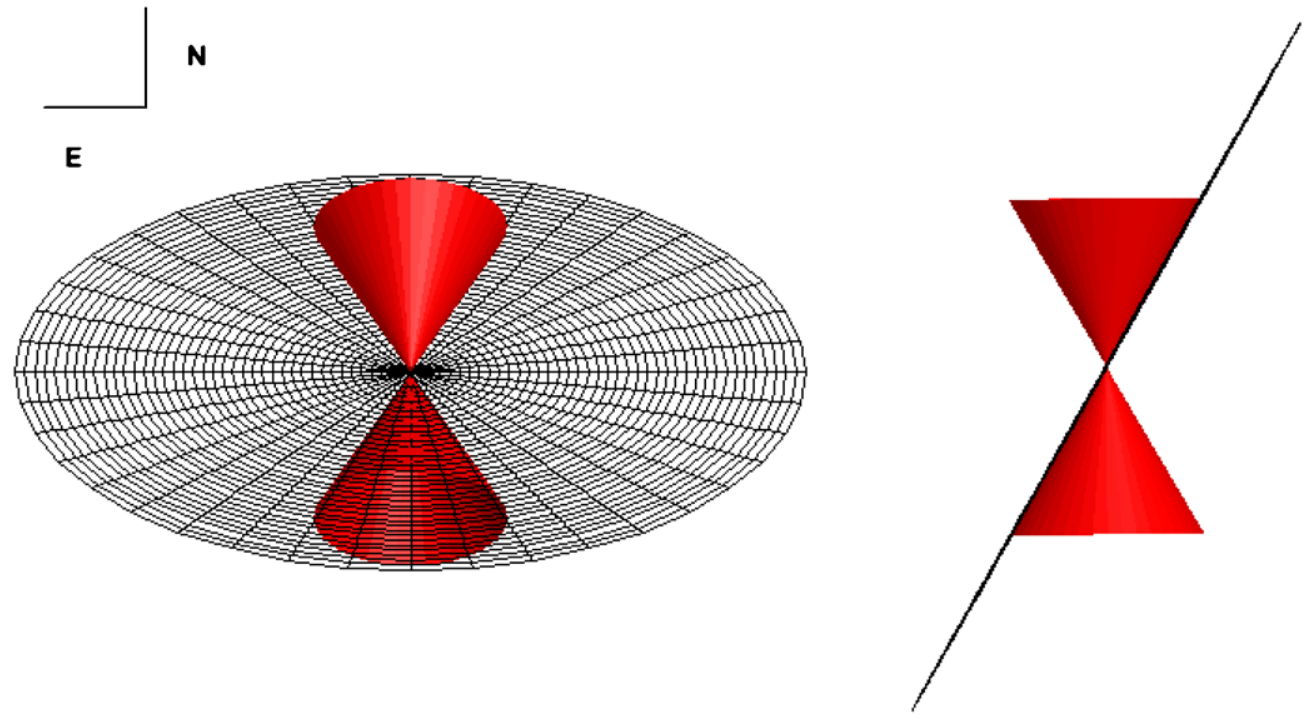

Figure 7. Test model of a hypothetical Seyfert galaxy with the NLR and disk parameters given in Table 2. The left-hand side shows our view, and the right hand side shows a view in which the bicone axis is in the plane of the sky and the galactic disk is edge-on (our view is to the lower left and in the page).

(A color version of this figure is available in the online journal.) 
Are there cases where a combination of outflows and extinction can lead to a redshifted emission line? The answer is yes. In Figure 7, we show a test case, based on the parameters listed in Table 2. In this extreme case, the P.A. and inclination of the galactic disk are such that the blueshifted cone in the south is entirely occulted by the disk and the redshifted cone in the north is not. To investigate this issue further, we ran a simulation for every possible combination of disk inclination, bicone inclination, and difference in P.A. in $1^{\circ}$ intervals, weighted by the probability of observing a disk or bicone at a particular inclination $(\propto \sin i)$. Assuming a random distribution of these parameters, we find that the percentage of the total population that shows more extinction of the redshifted portion of the bicone than the blueshifted portion is $17.2 \%, 16.7 \%, 15.6 \%$, and $13.7 \%$ for H.O.As. of the bicone of $30^{\circ}, 40^{\circ}, 50^{\circ}$, and $60^{\circ}$, respectively. Thus, this model can explain the relatively small number of Seyfert galaxies that show redshifted emission lines. However, the above percentages cannot be directly compared to the observed values because the observational errors do not allow us to determine the fraction of Seyferts that have redshifted velocities between 0 and $50 \mathrm{~km} \mathrm{~s}^{-1}$. Ideally, one could attempt to match the distribution in Figure 1 by generating emission line profiles from more realistic models that include thick disks and bicones, a proper treatment of the dust extinction as a function of position, realistic velocities and dispersions as a function of position, and a more sophisticated error model, but that type of simulation is beyond the scope of this paper.

\section{SUMMARY AND CONCLUSIONS}

We find that $\sim 40 \%$ of Seyfert galaxies show radial velocity offsets $|\Delta v| \geqslant 50 \mathrm{~km} \mathrm{~s}^{-1}$ between their narrow emission lines and host galaxy lines. The distribution of offsets peaks near $0 \mathrm{~km} \mathrm{~s}^{-1}$, but has a strong blue tail extending up to $\sim-250 \mathrm{~km} \mathrm{~s}^{-1}$; only $\sim 6 \%$ of the Seyferts in the sample show significantly redshifted emission lines, up to $100 \mathrm{~km} \mathrm{~s}^{-1}$. The Seyfert 1 distribution is shifted to higher blueshifts on average.

To investigate the nature of the velocity offsets, we relied on our previous investigations into the reddening, physical conditions, and kinematics of the NLRs in NGC 1068 (Seyfert 2) and NGC 4151 (Seyfert 1), which were based on spatially resolved $H S T$ /STIS spectra and phototionization and kinematic models. The parameters from these studies allowed us to generate geometric models of the biconical outflow in the NLR and extinction by dust in the inner galactic disk. We also collapsed the high-resolution HST/STIS spectra obtained at multiple slit locations to simulate ground-based observations. The resulting [O III] emission-line profiles are asymmetric to the blue and have blue-shifted centroids, which can be attributed to preferential extinction of the redshifted clouds in the geometric models of these two Seyferts.

It is clear from the above examples that models that incorporate biconical outflow and extinction by the inner galactic disk are more likely to produce blueshifted velocity centroids than redshifted ones, because the blueshifted clouds are closer to the observer and less likely to be extincted in most cases. However, we have shown that redshifted velocity centroids are possible in certain cases, for example when the disk is highly inclined and covers most of the near cone. Furthermore, one would expect from these models that more Seyfert 1s would show blueshifts than Seyfert 2s, because the near cone would be pointed more directly at us and therefore less extincted on average. All of these features of the geometric models are in agreement with the observed velocity offsets. However, we note that the [O III] emission in NGC 1068 shows a higher blueshift than that in NGC 4151, which indicates that individual variations in velocities and extinctions of the NLR clouds amongst AGN are important in determining the overall distribution in velocity offsets.

We conclude that the velocity offsets of emission lines in low$z$ Seyfert galaxies are primarily due to a combination of radial outflow in their NLRs and extinction by dust on the same size scale as their NLRs. More exotic explanations are not required. Furthermore, we find that the dust responsible for the extinction must be primarily exterior to the ionized gas. The dust features seen in the inner kpc of Seyfert galaxies, mostly in the form of dust spirals (Martini et al. 2003, and references therein), are the likely culprits.

As we have already noted, the bumps and peaks in the emission-line profiles are due to large bright knots of emissionline gas traveling at their own peculiar velocities, on top of the general outflow pattern. Thus, another contributor to the velocity offsets could be an uneven distribution of knots in velocity space in an AGN, resulting in more (or brighter) blueshifted knots than redshifted ones, or vice versa. This effect alone, however, will not lead to an asymmetric distribution of velocity offsets.

It is instructive to compare the distributions of velocity offsets between narrow emission and stellar absorption lines in the low $z(<0.043$, Nelson \& Whittle 1995) and moderate $z(0.34<z<$ 0.92, Comerford et al. 2009) samples. The magnitudes of the velocity offsets in the two distributions are essentially the same, and they have a similar percentage of "significant" $\left(\gtrsim 50 \mathrm{~km} \mathrm{~s}^{-1}\right)$ offsets $(41 \%$ at low $z, 35 \%$ at moderate $z)$. However, the distribution for the moderate $z$ sample is roughly symmetric around $0 \mathrm{~km} \mathrm{~s}^{-1}$, which is surprising, because these AGNs are likely to have outflows in their NLRs as well. In fact, Comerford et al. use the symmetric distribution as a reason to disfavor the outflows plus dust explanation and favor the inspiralling black hole scenario. This, in turn, leads them to a merger fraction of $\sim 30 \%$ for red galaxies at these redshifts, which is a surprising large number, particularly since these galaxies show no evidence for star formation.

The nature of the Comerford et al. (2009) sample provides a couple of possible explanations for a symmetric distribution of velocity offsets in the context of mass outflows. Their sample contains only Seyfert 2 galaxies that have been selected to have red host galaxies, to specifically avoid contamination from ionized gas around star-forming regions. The lack of significant star formation suggests a lack of dusty gas, and therefore a lack of extinction, in their NLRs. The velocity offsets would therefore be dominated by uneven distributions of emission-line knots in at least some of the AGNs, with no preference for blueshifted profiles. Another possible explanation is that the red colors are due to highly inclined disk galaxies, which, as shown in Figure 7, will lead to a significant number of redshifts and a more symmetric distribution of velocity offsets. Nevertheless, there is a local example of velocity (and spatial) offsets due to multiple AGNs in the nearby galaxy NGC 3341 (Barth et al. 2008), and one might expect more of these at higher redshifts from galaxy mergers. However, the emission-line ratios in NGC 3341 indicate significant star formation, as might be expected from mergers, and if it was at the appropriate redshift, NGC 3341 would have not been included in the Comerford et al. sample. Both the low $-z$ and moderate- $z$ samples are rather small; larger sample would be helpful in clarifying the role that mergers might play in producing velocity offsets. 
Finally, we note that ground-based spectra of local Seyfert galaxies, particularly those obtained at high spectral resolution (Vrtilek \& Carleton 1985; Veilleux 1991b; Nelson \& Whittle 1995), show a wide variety of narrow emission-line profiles. The profiles are often very irregular in appearance, with bumps or shelves in their red or blue wings, and a number of them show multiple peaks, like those in NGC 1068 (Figure 4), due to the contributions of distinct emission-line knots. Of particular interest is the [O III] profile of Mrk 78 (Vrtilek \& Carleton 1985; Whittle et al. 1988; Nelson \& Whittle 1995), which shows two large well-defined peaks separated by $\sim 800 \mathrm{~km} \mathrm{~s}^{-1}$, and yet there is no evidence for displaced or double SMBHs in Mrk 78. This velocity separation is actually larger than those in the two "dual AGNs" of Comerford et al. (2009), which show velocity separations between double-peak profiles of $630 \mathrm{~km} \mathrm{~s}^{-1}$ and $440 \mathrm{~km} \mathrm{~s}^{-1}$. Thus, even these double-peaked AGNs may not require the presence of double SMBHs.

This research has made use of the NASA/IPAC Extragalactic Database (NED) which is operated by the Jet Propulsion Laboratory, California Institute of Technology, under contract with the National Aeronautics and Space Administration. This research has made use of NASA's Astrophysics Data System Bibliographic Services.

\section{REFERENCES}

Antonucci, R. 1993, ARA\&A, 31, 473

Arribas, S., Mediavilla, E., \& García-Lorenzo, B. 1996, ApJ, 463, 509

Axon, D. J., Marconi, A., Capetti, A., Maccetto, F. D., Schreier, E., \& Robinson, A. 1998, ApJ, 496, L75

Baker, J. G., et al. 2008, ApJ, 682, L29

Barbosa, F. K. B., Storchi-Bergmann, T., Fernandes, R. C., Winge, C., \& Schmitt, H. 2009, MNRAS, 396, 2

Barth, A. J., Bentz, M. C., Greene, J. E., \& Ho, L. C. 2008, ApJ, 683, L119

Bian, W., Yuan, Q., \& Zhao, Y. 2005, MNRAS, 364, 187

Bogdanović, T., Eracleous, M., \& Sigurdsson, S. 2009, ApJ, 697, 288

Boroson, T. 2005, ApJ, 130, 381

Bottinelli, L., Gouguenheim, L., Fouque, P., \& Paturel, G. 1990, A\&AS, 82, 391

Busco, I. C., \& Steiner, J. E. 1992, MNRAS, 258, 306

Campanelli, M., Lousto, C., Zlochower, Y., \& Merritt, D. 2007, Phys. Rev. Lett., 98,231102

Capetti, A., Axon, D. J., Macchetto, F. D., Marconi, A., \& Winge, C. 1999, ApJ, 516, 187

Capriotti, E., Foltz, C., \& Byard, P. 1979, ApJ, 230, 681

Cecil, G., Dopita, M. A., Groves, B., Wilson, A. S., Ferruit, P., Pecontal, E., \& Binette, L. 2002, ApJ, 568, 627

Christopoulou, P. E., Holloway, A. J., Steffen, W., Mundell, C. G., Thean, A. H. C., Goudis, C. D., Meaburn, J., \& Pedlar, A. 1997, MNRAS, 284, 385

Comerford, J. M., et al. 2009, ApJ, 698, 956

Corbin, M. R. 1990, ApJ, 357, 346

Crenshaw, D. M. 1986, ApJS, 62, 821

Crenshaw, D. M., \& Kraemer, S. B. 2000, ApJ, 532, L101

Crenshaw, D. M., Kraemer, S. B., Hutchings, J. B., Bradley, L. D., II, Gull, T. R., Kaiser, M. E., Nelson, C. H., Ruiz, J. R., \& Weistrop, D. 2000, AJ, 120,1731

Dahari, O., \& De Robertis, M. M. 1988, ApJ, 331, 727

Das, V., Crenshaw, D. M., Hutchings, J. B., Deo, R. P., Kraemer, S. B., Gull, T. R., Kaiser, M. E., Nelson, C. H., \& Weistrop, D. 2005, AJ, 130, 945
Das, V., Crenshaw, D. M., \& Kraemer, S. B. 2007, ApJ, 656, 699

Das, V., Crenshaw, D. M., Kraemer, S. B., \& Deo, R. P. 2006, AJ, 132, 620

Deo, R. P., Crenshaw, D. M., \& Kraemer, S. B. 2006, AJ, 132, 321

De Robertis, M. M., \& Osterbrock, D. E. 1986, ApJ, 301, 727

De Robertis, M. M., \& Shaw, R. A. 1990, ApJ, 348, 421

de Vaucouleurs, G., de Vaucouleurs, A., Corwin, H. G. Jr., Buta, R. J., Paturel, G., \& Fouqu, P. 1991, in Third Reference Catalogue of Bright Galaxies (New York: Springer) (RC3)

Dotti, M., Montuori, C., Decarli, R., Volonteri, M., Colpi, M., \& Haardt, F. 2009, MNRAS, 398, L73

Gaskell, C. M. 1982, ApJ, 263, 79

Heckman, T. M., Krolik, J. H., Moran, S. M., Schnittman, J., \& Gezari, S. 2009, ApJ, 695, 363

Heckman, T. M., Miley, G. K., van Breugel, W. J. M., \& Butcher, H. R. 1981, ApJ, 247, 403

Hutchings, J. B., et al. 1998, ApJ, 492, L115

Komossa, S., Xu, D., Zhou, H., Storchi-Bergmann, T., \& Binette, L. 2008a, ApJ, 680,926

Komossa, S., Zhou, H., \& Lu, H. 2008b, ApJ, 678, L81

Kraemer, S. B., \& Crenshaw, D. M. 2000, ApJ, 544, 763

Kraemer, S. B., Crenshaw, D. M., Hutchings, J. B., Gull, T. R., Kaiser, M. E., Nelson, C. H., \& Weistrop, D. 2000, ApJ, 531, 278

Malkan, M. A., Gorjian, V., \& Tam, R. 1998, ApJS, 117, 25

Martini, P., \& Pogge, R. W. 1999, AJ, 118, 2646

Martini, P., Regan, M. W., Mulchaey, J. S., \& Pogge, R. W. 2003, ApJ, 589, 774

Moore, D., Cohen, R. D., \& Marcy, G. W. 1996, ApJ, 470, 280

Nelson, C. H., \& Whittle, M. 1995, ApJS, 99, 67

Netzer, H. 1977, MNRAS, 181, 89p

Osterbrock, D. E. 1981, ApJ, 246, 697

Osterbrock, D. E., \& Cohen, R. 1979, MNRAS, 187, 61p

Osterbrock, D. E., \& Mathews, W. G. 1986, ARA\&A, 24, 171

Pelat, D., Alloin, D., \& Fosbury, R. A. E. 1981, MNRAS, 195, 787

Penston, M. V. 1977, MNRAS, 180, 27p

Pogge, R. W., \& Martini, P. 2002, ApJ, 569, 624

Regan, M. W., \& Mulchaey, J. S. 1999, AJ, 116, 2676

Richards, G. T., et al. 2002, AJ, 124, 1

Riffel, R. A., Storchi-Bergmann, T., Dors, O. L., \& Winge, C. 2009, MNRAS, 393, 783

Rodríguez-Ardila, A., Prieto, M. A., Viegas, S., \& Gruenwald, R. 2006, ApJ, 653,1098

Ruiz, J., Crenshaw, D. M., Kraemer, S. B., Bower, G. A., Gull, T. R., Hutchings, J. B., Kaiser, M. E., \& Weistrop, D. 2001, AJ, 122, 2961

Savage, B. D., \& Mathis, J. S. 1979, ARA\&A, 17, 73

Schlesinger, K., Pogge, R. W., Martini, P., Shields, Joseph C., \& Fields, D. 2009, ApJ, 699, 857

Shields, G. A., Bonning, E. W., \& Saliander, S. 2009, ApJ, 696, 1367

Shull, J. M., \& van Steenberg, M. E. 1985, ApJ, 294, 599

Snedden, S. A., \& Gaskell, C. M. 2007, ApJ, 669, 126

Stoklasová, I., Ferruit, P., Emsellem, E., Jungwiert, B., Pécontal, E., \& Sánchez, S. F. 2009, A\&A, 500, 1287

Storchi-Bergmann, T., Simões-Lopes, R. D., McGregor, P. J., Riffel, R. A., Beck, T., \& Martini, P. 2009, MNRAS, in press (arXiv:0911.2212)

Storchi-Bergmann, T., Wilson, A. S., \& Baldwin, J. A. 1992, ApJ, 396, 45

Sulentic, J. W., Marziani, P., \& Dultzin-Hacyan, D. 2000, ARA\&A, 38, 521

Tytler, D., \& Fan, X. 1992, ApJS, 79, 1

Veilleux, S. 1991a, ApJ, 369, 331

Veilleux, S. 1991b, ApJS, 75, 383

Veilleux, S. 1991c, ApJS, 75, 357

Vrtilek, J. M., \& Carleton, N. P. 1985, ApJ, 294, 106

Whittle, M. 1985, MNRAS, 213, 1

Whittle, M., Pedlar, A., Meurs, E. J. A., Unger, S. W., Axon, D. J., \& Ward, M. J. 1988, ApJ, 326, 125

Wilkes, B. J. 1984, MNRAS, 207, 73

Xilouris, E. M., Byun, Y. I., Kylafis, N. D., Paleologou, E. V., \& Papamastorakis, J. 1999, A\&A, 344, 868 